\title{
Stereotactic EEG via multiple single-path omnidirectional trajectories within a single platform: institutional experience with a novel technique
}

\author{
Michael C. Dewan, MD, MSCI, ${ }^{1}$ Robert Shults, BS, ${ }^{2}$ Andrew T. Hale, MS, ${ }^{1}$ Vishad Sukul, MD, ${ }^{1}$ \\ Dario J. Englot, MD, PhD, ${ }^{1}$ Peter Konrad, MD, PhD, ${ }^{1}$ Hong Yu, MD, ${ }^{1}$ Joseph S. Neimat, MD, ${ }^{3}$ \\ William Rodriguez, MEd, ${ }^{2}$ Benoit M. Dawant, PhD, ${ }^{2}$ Srivatsan Pallavaram, PhD, ${ }^{2}$ and \\ Robert P. Naftel, MD1 \\ 1Department of Neurological Surgery, Vanderbilt University Medical Center; '2Department of Engineering and Computer Science, \\ Vanderbilt University, Nashville, Tennessee; and ${ }^{3}$ Department of Neurological Surgery, University of Louisville, Kentucky
}

OBJECTIVE Stereotactic electroencephalography (SEEG) is being used with increasing frequency to interrogate subcortical, cortical, and multifocal epileptic foci. The authors describe a novel technique for SEEG in patients with suspected epileptic foci refractory to medical management.

METHODS In the authors' technique, standard epilepsy evaluation and neuroimaging are used to create a hypothesisdriven SEEG plan, which informs the 3D printing of a novel single-path, multiple-trajectory, omnidirectional platform. Following skull-anchor platform fixation, electrodes are sequentially inserted according to the preoperative plan. The authors describe their surgical experience and technique based on a review of all cases, adult and pediatric, in which patients underwent invasive epilepsy monitoring via SEEG during an 18-month period at Vanderbilt University Medical Center. Platform and anatomical variables influencing localization error were evaluated using multivariate linear regression.

RESULTS Using this novel technology, 137 electrodes were inserted in 15 patients with focal epilepsy with favorable recording results and no clinical complications. The mean entry point localization error was $1.42 \mathrm{~mm}$ (SD $0.98 \mathrm{~mm}$ ), and the mean target point localization error was $3.36 \mathrm{~mm}(\mathrm{SD} 2.68 \mathrm{~mm}$ ). Platform distance, electrode trajectory angle, and intracranial distance, but not skull thickness, were independently associated with localization error.

CONCLUSIONS The multiple-trajectory, single-path, omnidirectional platform offers satisfactory accuracy and favorable clinical results, while avoiding cumbersome frames and prohibitive up-front costs associated with other SEEG technologies.

https://thejns.org/doi/abs/10.3171/2017.6.JNS17881

KEY WORDS epilepsy; frameless; platform; omnidirectional; SEEG; stereotactic electroencephalography; surgical technique; diagnostic technique

$\Lambda$ CCURATE localization of epileptogenic foci is the fundamental tenet of preoperative evaluation for surgical epilepsy. ${ }^{11}$ Traditionally, monitoring with scalp electrodes,$^{10}$ MRI,, 5 positron emission tomography (PET) ${ }^{13}$ neuropsychological testing, ${ }^{8}$ and analysis of seizure semiology have been the primary means of noninvasive diagnosis and evaluation. Invasive monitoring with subdural grids and strips offers finer resolution of the epileptogenic zone (EZ), particularly in patients with a superficial, cortical focus. However, in patients without a superficial onset, subdural electrodes may provide inadequate localization to permit a safe and effective resection. Stereotactic electroencephalography (SEEG) allows for interrogation of deeper structures and epileptic networks.

SEEG electrodes were originally inserted using a Talairach frame. Techniques have evolved to using stereotactic

ABBREVIATIONS DBS = deep brain stimulation; EPLE = entry point localization error; EZ = epileptogenic zone; IQR = interquartile range; $M E G=$ magnetoencephalography; $\mathrm{PET}$ = positron emission tomography; SEEG = stereotactic electroencephalography; TPLE = target point localization error.

SUBMITTED April 7, 2017. ACCEPTED June 13, 2017.

INCLUDE WHEN CITING Published online December 15, 2017; DOI: 10.3171/2017.6.JNS17881. 
frames, frameless stereotaxy, and robotic insertion; each of these techniques carries its own set of limitations, including prolonged operative time, accuracy shortcomings, and prohibitive device costs, respectively. In this report we describe a novel technique for implanting SEEG electrodes utilizing a personalized platform outfitted with a multitude of individual trajectories for complex electrode insertion. This technology provides an alternative method of implanting electrodes accurately, safely, and efficiently, while avoiding cumbersome traditional frames or expensive infrastructure, such as a robot. Our institutional experience with planning, manufacturing, and electrode implantation is described, along with a detailed analysis of device-related and anatomical contributors to electrode accuracy.

\section{Methods \\ Patients}

All patients, pediatric and adult, at Vanderbilt University Medical Center undergoing SEEG utilizing the FHC microTargeting Multi-Oblique Epilepsy Platform (FHC, Inc.) from January 2015 to June 2016 were included in this study. Four surgeons performed these procedures. Patients were recommended for invasive monitoring after being diagnosed with refractory epilepsy and undergoing a noninvasive epilepsy evaluation that included a combination of continuous video EEG, MRI, PET, magnetoencephalography (MEG), single photon emission computed tomography (SPECT), neuropsychological testing, and/or functional MRI, and in some cases also an intracarotid sodium amobarbital procedure (Wada test). After completion of this evaluation, patients were discussed at a multidisciplinary surgical epilepsy conference that included members of the neurology, neurosurgery, radiology, and neuropsychology services. The presentation resulted in hypotheses about the EZ. Patients who were identified as having an EZ that was deep, possibly bilateral, or a part of an epileptic network and those with a history of failed subdural grid evaluation were considered candidates for SEEG. A hypothesis-driven SEEG implantation plan using a combination of orthogonal and oblique trajectories, as indicated, was formulated.

The workflow is described in detail below. In summary, the SEEG implantation plan is converted into trajectory plans on images from the stereotactic MRI study. This can be performed at any point before surgery after the stereotactic MRI. One week prior to electrode implantation, patients undergo bone fiducial anchor (WayPoint Fiducial Anchors; FHC, Inc.) placement and stereotactic CT. The stereotactic CT study is co-registered with the stereotactic MRI study, including the planned trajectories. The co-registered plan is then used for 3D printing of the platform. On the date of the implantation, the platform is fixed to the bone fiducial anchors and the electrodes are implanted as described.

\section{Trajectory Planning}

Stereotaxis is based on a thin-cut MRI study performed within 3 months of surgery and including T1-weighted sequences (with and without contrast), FLAIR and T2- weighted sequences, and MR angiography; in some cases MR venography and/or CT venography may also be performed. MR sequences and MEG or functional MRI data are co-registered within WayPoint Navigator software (FHC, Inc.). Next, patient anatomy is identified to generate the anterior commissure-posterior commissure (AC-PC) line, providing orientation for trajectory planning and an accuracy resolution of approximately $1 / 100$ th of a millimeter. ${ }^{7,18}$ Once registered, the previously agreed-upon hypothesis-driven SEEG implantation plan is converted into electrode trajectories within the software. Trajectories are formed by choosing an entry point and a target point. Depending on surgeon preference, the trajectories are planned in different display orientations, including AC-PC view, trajectory view, or the original MRI orientation. To expedite the process for multiple-trajectory planning, there are functions that allow trajectory cloning (for ipsilateral parallel trajectories) and mirroring (for contralateral trajectories). After all trajectories are planned, each is evaluated for risk of vascular injury with a specific emphasis on the entry site. Plans are adjusted as needed to avoid intersection with an artery or vein at the entry site.

Once all trajectories are finalized, the software conducts an automated simulation of electrode implantation at each trajectory. Preprogrammed electrode dimensions are available from a multitude of electrode manufacturers. During implantation simulation, the surgeon may visualize the actual coverage that can be expected. The electrode length - or depth of insertion-is easily adjusted to ensure complete and accurate coverage.

\section{Bone Fiducial Anchor Insertion}

Approximately 1 week prior to lead insertion, patients are fitted with bone fiducial anchors. The bone fiducial anchors are screwed into the skull and act as localizers for construction of the platform and as fixation points for the platform at the time of electrode insertion. The number of fiducial bone anchors is variable and depends on the planned trajectories (range 4-6 anchors). Anchors are placed through 5-mm incisions using local or general anesthesia, depending on surgeon preference and patient factors. The locations of the bone fiducial anchors depend on the number and location of trajectories. A stereotactic CT scan is then obtained with the bone fiducial anchors in place, allowing co-registration with the stereotactic MRI. Patients are discharged home the same day.

\section{Platform Manufacturing}

After co-registration, the stereotactic plan is then digitally uploaded to the manufacturer of the 3D platform (microTargeting Multi-Oblique Epilepsy Platform; FHC, Inc.). The manufacturer then creates the platforms and ships them to the hospital, a process that takes approximately 3-5 business days. Given the often bilateral nature and the diversity of sites and trajectories, the platform system may contain up to 3 separate pieces, each with at least 3 anchor pedestals (Fig. 1 left).

\section{Electrode Insertion}

Approximately 1 week after bone fiducial anchor place- 


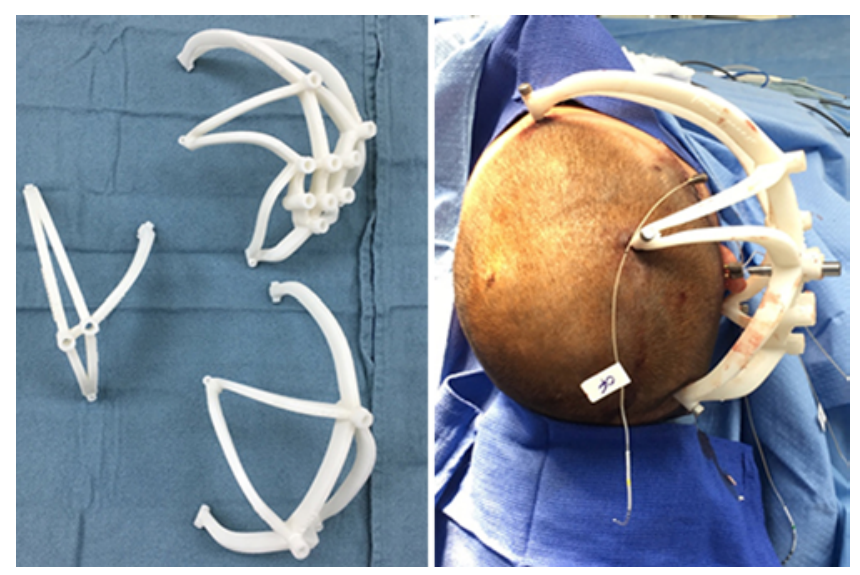

FIG. 1. Left: Three Starfix frames for a single patient requiring bilateral, multilobed interrogation. Right: A frame with 9 individual trajectories is affixed to the skull, and then electrodes are sequentially guided to their intracranial target. Figure is available in color online only.

ment, patients return to the operating suite for electrode insertion. After induction of general anesthesia, the patient's head is fixed in 3-point Mayfield pins if necessary and the scalp is prepared. The incisions overlying the bone fiducial anchors are opened and the anchors are exposed. The platform is then fixed to each of the bone fiducial anchors (Fig. 1 right). A drill guide is inserted into one of the stereotactic platform trajectories. A wrench is then inserted down the guide to mark the skin for a 3-mm incision. A high-speed drill bit is fixed with a depth stop for the skull thickness. The burr hole is then drilled through the platform instrument guide. Next, an obturator is advanced down the tool guide to assess the dural integrity. If the dura was not incidentally opened with the drill, then an endoscopic monopolar is inserted and the dura is opened with cautery.

The workflow from this point forward varies depending on the electrode manufacturer. If the surgeon prefers an anchor bolt, as available for Adtech or PMT electrodes, then the bolt wrench is passed down the tool guide and used to screw the anchor bolt directly into the hole for stereotactic electrode insertion. An obturator with a depth stop is passed down the bolt a distance premeasured from the platform plan. After reaching the target, the obturator is withdrawn, and in a similar fashion, an electrode with a premeasured depth stop is advanced along the trajectory through the anchor bolt. The electrode is secured with a watertight twist cap, the depth stop is removed, and the electrode is allowed to hang freely. The same sequence is repeated for all trajectories.

If Integra (Integra Life Sciences) electrodes are used (a decision based on surgeon preference only), a cannula-guided approach is used. The cannula and electrode depths for each trajectory are calculated and marked using depth stops. After the dura has been punctured as described above, reducing tubes are inserted through the drill guides to allow the cannula to be advanced to the desired depth and locked in place. The inner stylet of the cannula is removed. The electrode, which is held by an additional frame attachment, is then passed through the can- nula and secured in place at the desired depth. The outer cannula is then withdrawn while the electrode remains held in place at the intended target. The electrode inner stylet is removed, and the electrode is secured to the skin using a nylon suture prior to disassembly of the platform attachment.

After successful insertion of all electrodes, they are tested with electrocorticography to confirm successful recording. The platform and bone fiducial anchors are removed, and the bone fiducial anchor incisions are closed with absorbable sutures. Patients are then allowed to emerge from anesthesia and transferred to the intensive care or epilepsy monitoring unit, depending on the surgeon's practice pattern. A postoperative stereotactic CT scan is obtained to establish the location of each electrode. This postoperative CT is co-registered with the preoperative plan in the planning software, and the accuracy of the electrode placement is assessed.

\section{Error Measurement and Statistical Analysis}

To assess the accuracy and limitations of this implantation technique, the electrode trajectories and terminal locations displayed by the postimplantation stereotactic CT were compared with the preoperative plan. The Euclidean distance between the 2 was recorded both for entry point localization error (EPLE) and target point localization error (TPLE), as described in detail elsewhere. ${ }^{5}$ The entry point was defined as the point at which the trajectory passes through the inner cortical skull surface. The target point was defined as the planned termination of the electrode trajectory. The angle of lead implantation was defined as the number of degrees off the $90^{\circ}$ skull orthogonal. The platform-to-skull distance was defined as the linear measurement between the bottom surface of the platform and the skull surface. The skull thickness was measured along the trajectory of the electrode. The intracranial distance was measured from the inner cortical skull surface to the target point.

Localization errors were summarized as mean values with standard deviations, while non-normally distributed frame characteristics were described as medians with interquartile ranges (IQRs). Univariate linear regression was used to compare lead and anatomical characteristics against both localization errors. Measureable factors influencing localization error were sought. Parameters found to be significant $(\mathrm{p}<0.05)$ were incorporated into a multivariate linear regression model to identify independent factors responsible for localization error. All analyses and graph construction were performed with Stata version 14 (StataCorp).

\section{Results}

The institutional experience includes 15 patients and 137 intracranial leads (mean 9 leads per patient) (Table 1). An average of 3 lobes were interrogated per patient (range 2-7 lobes). Six patients underwent bilateral electrode implants. The average operative time was 207 minutes, and the average of the operative times per lead was 24 minutes. The mean EPLE was $1.42 \mathrm{~mm}$ (SD 0.98) and the mean TPLE was $3.36 \mathrm{~mm}$ (SD 2.68). There were no major com- 
TABLE 1. Patient and lead characteristics

\begin{tabular}{cclcccc}
\hline Case No. & Age (yrs), Sex & Laterality & No. of Lobes & No. of Leads & Operative Time (mins) & Operative Time per Lead (mins) \\
\hline 1 & $59, \mathrm{~F}$ & Unilateral & 2 & 5 & 175 & 35 \\
\hline 2 & $65, \mathrm{M}$ & Unilateral & 2 & 6 & 224 & 37 \\
\hline 3 & $24, \mathrm{M}$ & Bilateral & 2 & 8 & 222 & 28 \\
\hline 4 & $21, \mathrm{M}$ & Bilateral & 7 & 8 & 221 & 28 \\
\hline 5 & $39, \mathrm{M}$ & Unilateral & 2 & 8 & 237 & 22 \\
\hline 6 & $37, \mathrm{~F}$ & Bilateral & 2 & 11 & 165 & 21 \\
\hline 7 & $49, \mathrm{~F}$ & Unilateral & 2 & 8 & 278 & 35 \\
\hline 8 & $20, \mathrm{M}$ & Unilateral & 2 & 8 & 151 & 22 \\
\hline 9 & $32, \mathrm{~F}$ & Unilateral & 2 & 7 & 183 & 175 \\
\hline 10 & $27, \mathrm{M}$ & Bilateral & 4 & 9 & 194 & 19 \\
\hline 11 & $23, \mathrm{M}$ & Unilateral & 2 & 9 & 216 & 19 \\
\hline 12 & $45, \mathrm{M}$ & Bilateral & 6 & 10 & 174 & 17 \\
\hline 13 & $17, \mathrm{M}$ & Bilateral & 4 & 13 & 278 & 19 \\
\hline 14 & $9, \mathrm{M}$ & Unilateral & 3 & 12 & & 19 \\
\hline 15 & $15, \mathrm{M}$ & Unilateral & 4 & 15 & & 19 \\
\hline
\end{tabular}

plications, and all patients had satisfactory EEG recording trials resulting in subsequent therapeutic procedures. In 4 patients, small extraaxial or subarachnoid hemorrhages were detected on routine postoperative CT. No patient experienced symptomatic implantation sequelae, and there was no surgical adverse event requiring lead removal or replacement.

The distance from the platform to the skull was variable for each trajectory, averaging $43.2 \mathrm{~mm}$ (IQR 36.4-50.7 $\mathrm{mm}$ ). Similarly, the angle of lead implantation through the bone was routinely not orthogonal. The angle off the or- thogonal averaged $25.3^{\circ}$ (IQR $15.3^{\circ}-35.5^{\circ}$ ). Both increasing distance from the platform to the skull (Fig. 2) and the increasing angle of implantation off of the orthogonal (Fig. 3) correlated with worsening EPLE (coefficient $>0, p<$ 0.05) (see Table 3). The intracranial electrode distance to the target was variable, averaging $41.7 \mathrm{~mm}$ (IQR 34.9-50.7 $\mathrm{mm})$. Increasing distance from the platform to the skull (Fig. 2), increasing angle of implantation off of the orthogonal (Fig. 3), and increasing intracranial electrode distance (Fig. 4) correlated with worsening TPLE (Table 2). Neither skull thickness nor the use of a bolt fixation device was

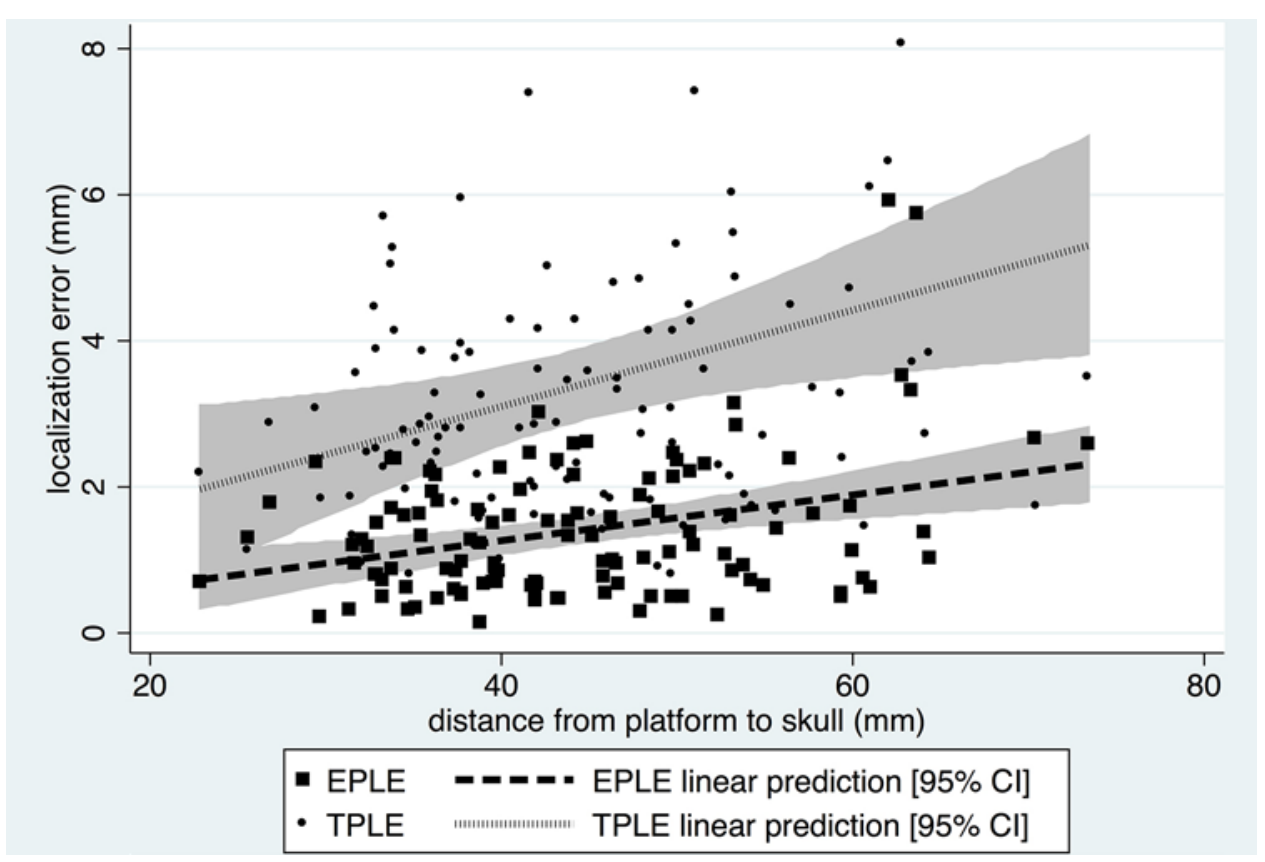

FIG. 2. The influence of platform distance on EPLE (squares) and TPLE (circles). The shaded area represents the 95\% confidence interval. Figure is available in color online only. 


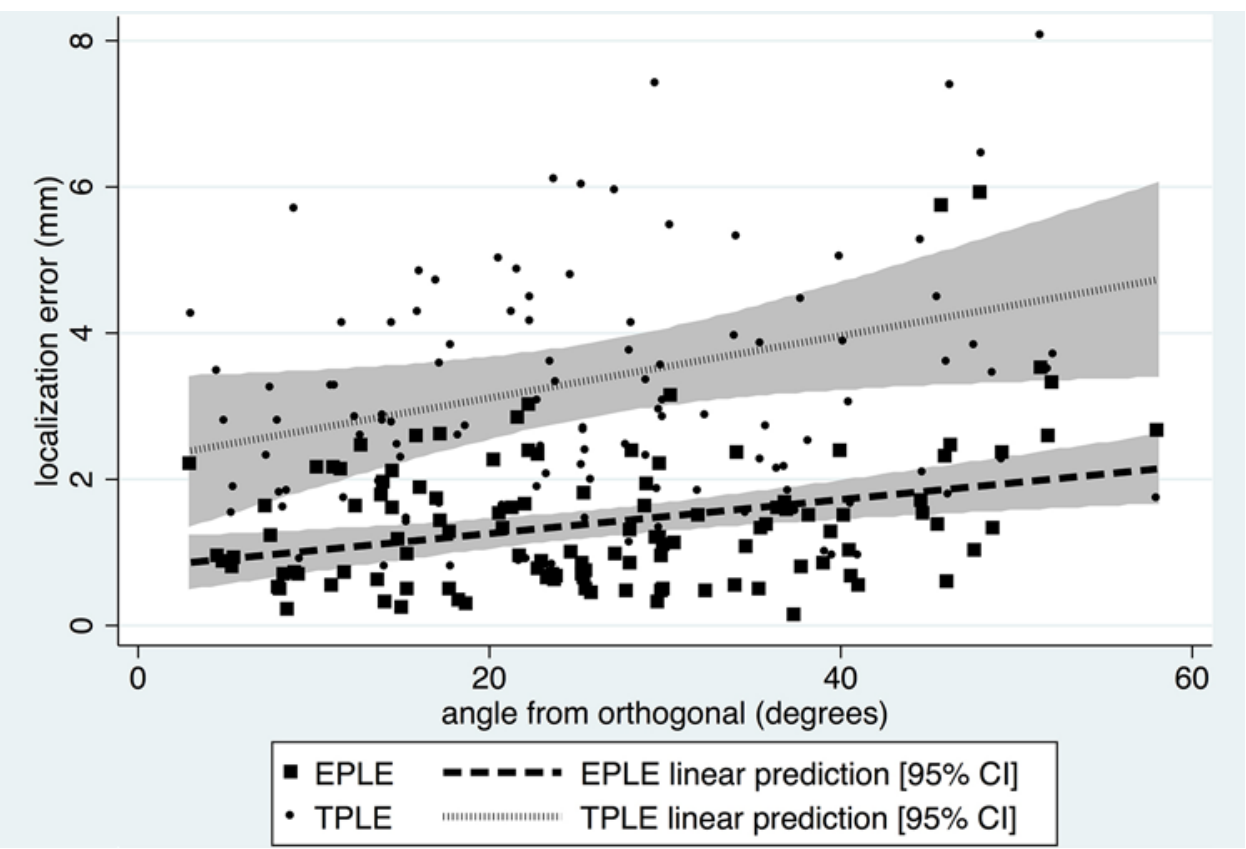

FIG. 3. The influence of insertion angle on EPLE (squares) and TPLE (circles). The shaded area represents the $95 \%$ confidence interval. Figure is available in color online only.

significantly associated with localization error $(p>0.05)$.

In the multivariate model, increasing distance from the platform and increasing angle of implantation from orthogonal were independent predictors of worsening EPLE (Table 3). The angle of implantation was found not to contribute significantly for TPLE. The remaining metrics, increasing distance from the platform and increas- ing intracranial distance, were independent predictors of increased TPLE.

Additionally, there was an interaction effect between platform distance and angle of implantation. As the angle from orthogonal increased, and as the distance between the platform and the skull increased, the associated increase in EPLE was observed to be greater than what

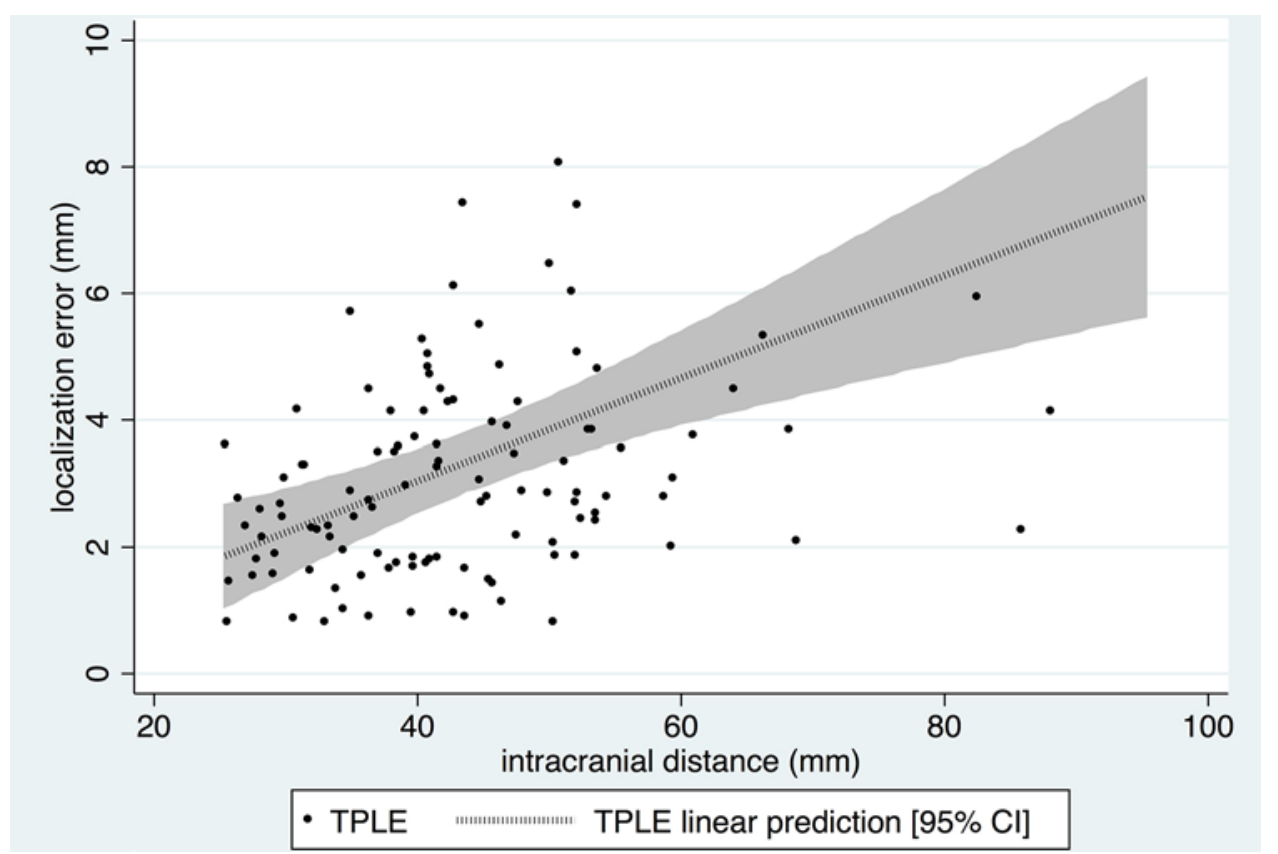

FIG. 4. The influence of intracranial distance on TPLE (circles). The shaded area represents the $95 \%$ confidence interval. Figure is available in color online only. 
TABLE 2. Univariate relationship between localization error and lead parameters

\begin{tabular}{|c|c|c|c|c|c|}
\hline \multirow[b]{2}{*}{ Covariate } & \multirow[b]{2}{*}{ Median (IQR) } & \multicolumn{2}{|c|}{ EPLE } & \multicolumn{2}{|c|}{ TPLE } \\
\hline & & Coef & $p$ Value & Coef & $p$ Value \\
\hline Platform-to-skull distance, mm & $43.17(36.4-50.7)$ & 0.0312 & $<0.001$ & 0.066 & 0.008 \\
\hline Skull thickness, mm & $6.7(5.2-8.9)$ & 0.041 & 0.196 & 0.076 & 0.393 \\
\hline Intracranial distance, $\mathrm{mm}$ & $41.7(34.9-50.7)$ & NA & NA & 0.08 & $<0.001$ \\
\hline Angle from orthogonal, ${ }^{\circ}$ & $25.3(15.3-35.5)$ & 0.023 & 0.001 & 0.043 & 0.03 \\
\hline Bolt (ref; no bolt) & NA & -0.01 & 0.964 & -0.32 & 0.551 \\
\hline
\end{tabular}

Coef = coefficient; NA = not applicable.

Boldface type indicates statistical significance.

would be expected from the sum of the individual effects. This interaction term-or synergism-itself was an independent predictor of error $(\mathrm{p}=0.004)$ and is illustrated via a heat map pictorially describing this relationship (Fig. 5).

\section{Discussion}

Herein, we describe our experience with the FHC microTargeting Multi-Oblique Epilepsy Platform outfitted with numerous, single-path trajectories to interrogate epileptic foci. To the best of our knowledge, this is the first description of a frameless, platform-based device utilizing individual trajectory guides for the insertion of multidirectional intracerebral electrodes in SEEG. We report a mean EPLE of $1.42 \mathrm{~mm}$ and a mean TPLE of $3.36 \mathrm{~mm}$, comparable to those of other non-robot-based technologies. ${ }^{17,19}$ The surgical goal was achieved in each case, and no clinical complications were observed. Additionally, we demonstrate the relationship between localization error and modifiable factors, including platform distance, insertion angle, and intracranial distance traversed.

For surgical treatment of epilepsy, the most important predictor of success is accurate localization of the EZ. ${ }^{6,15}$ While subdural grid electrodes provide the foundation for EEG evaluation of neocortical epilepsy, their utility diminishes with distance from the brain surface, and their feasibility wanes with bilateral or noncontiguous targets. SEEG, therefore, is valuable under several circumstances: a deep EZ, such as within the temporal lobe, cingulate gyrus, or insula; previous failure of or nondiagnostic subdural grid electrodes; and bilateral or otherwise noncontiguous candidate foci. 9,11

Accurate placement of intracerebral electrodes demands detailed anatomical mapping, both of the lesion and of the relevant structures to be avoided, and begins with precise co-registration of preoperative cranial imaging with fixed surgical anatomy. Generally, the SEEG platforms can be categorized into frame-based and frameless systems, each carrying its own advantages and disadvantages. ${ }^{6}$ Some frames support only multiple parallel and orthogonal trajectories, while others allow oblique trajectories capable of maneuvering about important neurovascular structures. ${ }^{1}$ Although reliable and accurate, frame-based methods may generate patient discomfort (especially in the pediatric population), consume valuable operative time, and limit the operative field. ${ }^{14,17}$

These drawbacks have provided the motivation for in- tracerebral electrode insertion using frameless technology. Robot-assisted SEEG is one such method that allows implantation of multiple electrodes in virtually any direction, providing the surgeon with unique flexibility. ${ }^{5}$ Surgical times can be truncated by eliminating the need for repeated frame coordinate adjustments. ${ }^{16}$ Additionally, intraoperative modifications to trajectories are permissible. ${ }^{10}$ However, a price tag ranging from hundreds of thousands of dollars to $\$ 2.5$ million for the device can deter adoption of this technology, particularly for modest-volume epilepsy centers. ${ }^{3}$ Maintenance of the technology and additional training required for some adaptable parts may also serve as barriers to a smooth transition to robot-assisted implantation.

While SEEG has undergone many refinements since its inception a half century ago, ${ }^{2}$ the approach described in this report offers some advantages relative to its predecessors. Its comparatively simple design combines conditional speed and versatility to deliver a surgeon-friendly experience with patient comfort as a central consideration. Patient inconvenience, however, must be taken into account, as this system mandates that patients visit the operating room twice-first for bone anchor placement and again for lead insertion. Because all lead trajectory planning is conducted prior to the date of surgical implantation, neither immediate preoperative nor intraoperative imaging is necessary. In a similar frameless design, Balanescu and colleagues report a 50\% reduction in the time to place a single electrode relative to the time required when using an analogous frame-based design. ${ }^{1}$ Time is also saved in the application of the platform, which typically can be done in less than a minute by securing the platform legs to corresponding bone anchors. We caution that while substantial operative time may be saved during the lead insertion phase, the preceding surgery to place bone markers is a relevant and essential event that must be carefully considered by the surgeon and patient. As with other frameless systems, the platforms have been specifically manufactured to fit precisely on the bone anchors. Accordingly, registration of the preoperative imaging to the stationary bone markers obviates the need for a cumbersome frame placement. Furthermore, the frameless designs means that the head need not remain in geospatial fixation throughout the lead insertion procedure, and the surgeon can move the patient's head about to optimize working angles. The FHC platform is particularly beneficial in cases involving pediatric patients ( 3 of our 15), 
TABLE 3. Relationship between localization error and lead parameters adjusted for covariates

\begin{tabular}{lccc}
\hline \multicolumn{1}{c}{ Covariate } & Coef & SE & p Value \\
\hline EPLE & & & \\
\hline Platform-to-skull distance & 0.027 & 0.0084 & $\mathbf{0 . 0 0 2}$ \\
\hline $\begin{array}{c}\text { Intracranial distance } \\
\text { Angle from orthogonal }\end{array}$ & 0.016 & 0.007 & $\mathbf{0 . 0 2 2}$ \\
\hline $\begin{array}{c}\text { NPLE } \\
\text { Platform-to-skull distance }\end{array}$ & 0.066 & 0.023 & $\mathbf{0 . 0 0 5}$ \\
\hline Intracranial distance & 0.08 & 0.018 & $<0.001$ \\
\hline Angle from orthogonal & 0.008 & 0.019 & 0.68 \\
\hline
\end{tabular}

Boldface type indicates statistical significance.

who may be less likely to tolerate rigid fixation around a frame.

Others have described a faster mean lead insertion time using different techniques. Balanescu and coauthors report an average lead insertion time of 14 minutes using their frameless design. ${ }^{1}$ Using a Leksell frame-based technology in a series of 122 patients, Gonzalez-Martinez et al. describe an average operative time of 107 minutes, or approximately 8 minutes per lead. ${ }^{11}$ Upon adoption of the robot at their institution, the operative time per lead changed only nominally to approximately 10 minutes. ${ }^{10}$ In contrast, in a series of 500 patients undergoing SEEG via robot-assisted implantation, Cardinale et al. suggest an average lead insertion time between 24 and 25 minutes, ${ }^{5}$ more closely reflecting our experience with the technique described here. However, it is difficult to draw many conclusions about insertion time from our study because the experience was spread across so many surgeons that the effect of a learning curve could not yet be realized.

As illustrated by this series, the FHC individual-trajectory platform can accommodate complex, multiple, and bilateral targets in the same patient, while avoiding important neural and vascular structures. There is no practical limit to the number of electrodes that can be implanted. Here we describe a patient receiving 15 electrodes, although more certainly could have been placed had there been a clinical need. The number of electrodes is determined on the basis of the pathology, and is not constrained by the platform. Beyond a certain number of electrodes, the evaluation of epileptic foci approaches more of a fishing expedition than a hypothesis-driven operation. This cohort also illustrates how omnidirectional electrode insertion can be achieved without sacrificing accuracy. With a mean EPLE of less than $1.5 \mathrm{~mm}$, our results fall within the acceptable ranges. Our mean TPLE is more than twice this Euclidean distance, at $3.36 \mathrm{~mm}$, but the reader is cautioned against concluding a discouraging result. Unlike other intracranial stereotactic procedures (deep brain stimulation [DBS], biopsy), EPLE-not TPLE-is the crucial measurement. It is at the entry point wherein most hemorrhagic complications occur, and placement errors can result in symptomatic hemorrhage. In SEEG, the trajectory, not the target point, determines recording success. In a recent editorial on the subject, Cardinale notes,

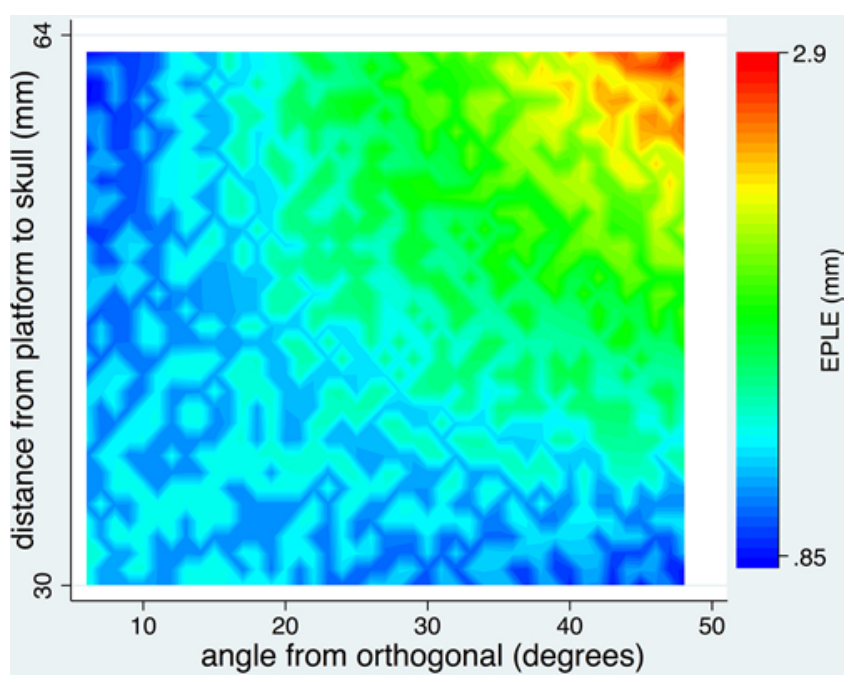

FIG. 5. The influence of platform distance and insertion angle on EPLE. The vertical legend on the right represents EPLE in millimeters. EPLE increases synergistically with distance and angle. Figure is available in color online only.

"The TP [target point] represents not a real target, but just the deepest point of the trajectory." Validation in a larger patient cohort is warranted, but our results indicate that an omnidirectional frameless platform can offer satisfactory stereotactic lead placement capabilities.

Finally, this technology can be easily adopted with relatively little capital investment by hospitals. Wherein a robot can cost millions, the WayPoint Navigator software and FHC platform are far more affordable, and they are compatible with most modern imaging technology. Moreover, similar frameless technology is already being used by neurosurgeons for implantation of DBS electrodes. ${ }^{12}$ Thus, familiarity with the technology for some functional neurosurgeons may ease the learning curve when applied to epilepsy patients requiring SEEG. Indeed, this was precisely our experience. We have found the software easy to use, the manufacturing punctual and precise, and the clinical results excellent. We are among the highest-volume DBS centers, and in DBS surgery submillimetric accuracy is essential, so transitioning to use of the FHC frame for SEEG was comfortable.

These advantages notwithstanding, there are important considerations that must be made before adopting the technique. Three limitations warrant specific elaboration. 1) Two separate procedures (bone anchor placement and electrode insertion) are required, whereas most framebased methods can be completed in just one. Naturally, the additional operating room time and inconvenience to the patient must be weighed. In our institutional experience with this technology (primarily for DBS), the bone anchor placement is quick and uniformly well tolerated. ${ }^{12} 2$ ) There is little forgiveness for manufacturing mishaps or interruptions. A defective platform design or shipping delay, for example, could conceivably cause postponement or cancellation of surgery. Essentially, an extraneous entity beyond the immediate control of the surgeon is introduced, leaving open a small, but plausible window for error. Among 
more than 1000 cases performed at our institution using this technology for DBS, 2 such instances have arisen. 3) Intraoperative adjustment of a given electrode trajectory is not possible. Each path is itself a rigid column through which the sheath, obturator, drill, and electrode all pass in sequence. As could be done with robot technology, finetuning the insertion point and angle with this technology is not permitted. Minor depth adjustments, meanwhile, are permissible and can be achieved without compromising the length-dependent recording feature of SEEG electrodes. An additional limitation also deserves comment: target point accuracy relative to robot-assisted techniques. In 2 large case series in which patients underwent robotassisted implantation, the TPLE was between 1.7 and 2.0 $\mathrm{mm} .{ }^{5,10}$ This may be compared with our TPLE of $3.36 \mathrm{~mm}$ and TLPEs in other frameless series $\left(3.66 \mathrm{~mm}^{17}\right.$ and 3.2 $\mathrm{mm}^{19}$ ). Finally, while start-up costs for the FHC platform are much more modest than those associated with the robot, hospitals must take into consideration the cost of the additional surgery for the former technique. While beyond the scope of this study, a formal cost-effective analysis in a larger patient cohort may help hospital administrators and surgeons determine which system best suits their longterm objectives.

Operative times are difficult to interpret in this case series, as there was significant variability depending on surgeon and experience. The limited number of cases per surgeon precluded a quantitative comparative analysis or reasonable detection of a learning curve.

Our results also suggest that relatively simple adjustments to the existing planning algorithms can improve accuracy. Based on initial experience, modifications are being made to account for the effects of platform-to-skull distance and angle from orthogonal. Efforts are made to reduce the platform-to-skull distance, although a minimum distance is required, depending on the electrode manufacturer and technique of insertion (bolt vs no bolt). Longer drill guides are also being used, resulting in less distance below the frame for drill flexing and scything. Additionally, although nonorthogonal angles from the bone surface are a necessity in SEEG, efforts are made in planning to avoid the extremes of oblique angles to prevent scything. We expect that the manufacturing alterations made based on these results will minimize localization error in our future practice.

Despite these limitations, the advantages offered by the omnidirectional platform make the technology a feasible alternative to traditional SEEG methods. Further comparison between cases in which patients undergo frame-based versus frameless SEEG (with and without robot assistance) may help determine which technology offers superior clinical results, economic output, and patient satisfaction. With the ultimate goal of seizure freedom, further efforts to create an instrument that maximizes efficiency while reducing costs and discomfort should be pursued.

\section{Conclusions}

We report on an institutional cohort of 15 patients with complex medically refractory epilepsy who underwent SEEG via a novel frameless technology. The localization accuracy, ease of use, and up-front cost profile depict the omnidirectional platform as a feasible and capable technology. Engineering efforts to lower the platform height and avoid the use of shallow entry angles may further minimize localization errors. Larger cohorts and the experience of other centers are needed to validate this technology before it is considered among the primary tools in the SEEG armamentarium.

\section{Acknowledgments}

Extraction of the SEEG leads from postoperative patient imaging data and computation of various metrics were performed using the CRAVE tools developed with support from NIH R01EB006136 and NIH R01NS095291.

\section{References}

1. Balanescu B, Franklin R, Ciurea J, Mindruta I, Rasina A, Bobulescu RC, et al: A personalized stereotactic fixture for implantation of depth electrodes in stereoelectroencephalography. Stereotact Funct Neurosurg 92:117-125, 2014

2. Bancaud J, Angelergues R, Bernouilli C, Bonis A, Bordas-Ferrer M, Bresson M, et al: Functional stereotaxic exploration (SEEG) of epilepsy. Electroencephalogr Clin Neurophysiol 28:85-86, 1970

3. Barbash GI, Glied SA: New technology and health care costs - the case of robot-assisted surgery. N Engl J Med 363:701-704, 2010

4. Cardinale F: Stereoelectroencephalography: application accuracy, efficacy, and safety. World Neurosurg 94:570571, 2016

5. Cardinale F, Cossu M, Castana L, Casaceli G, Schiariti MP, Miserocchi A, et al: Stereoelectroencephalography: surgical methodology, safety, and stereotactic application accuracy in 500 procedures. Neurosurgery 72:353-366, 2013

6. Cossu M, Cardinale F, Castana L, Citterio A, Francione $\mathrm{S}$, Tassi L, et al: Stereoelectroencephalography in the presurgical evaluation of focal epilepsy: a retrospective analysis of 215 procedures. Neurosurgery 57:706-718, 2005

7. D'Haese PF, Pallavaram S, Konrad PE, Neimat J, Fitzpatrick JM, Dawant BM: Clinical accuracy of a customized stereotactic platform for deep brain stimulation after accounting for brain shift. Stereotact Funct Neurosurg 88:81-87, 2010

8. Drane DL, Ojemann JG, Kim MS, Gross RE, Miller JW, Faught RE Jr, et al: Interictal epileptiform discharge effects on neuropsychological assessment and epilepsy surgical planning. Epilepsy Behav 56:131-138, 2016

9. Gonzalez-Martinez J, Bulacio J, Alexopoulos A, Jehi L, Bingaman W, Najm I: Stereoelectroencephalography in the "difficult to localize" refractory focal epilepsy: early experience from a North American epilepsy center. Epilepsia 54:323-330, 2013

10. González-Martínez J, Bulacio J, Thompson S, Gale J, Smithason S, Najm I, et al: Technique, results, and complications related to robot-assisted stereoelectroencephalography. Neurosurgery 78:169-180, 2016

11. Gonzalez-Martinez J, Mullin J, Vadera S, Bulacio J, Hughes G, Jones S, et al: Stereotactic placement of depth electrodes in medically intractable epilepsy. J Neurosurg 120:639-644, 2014

12. Konrad PE, Neimat JS, Yu H, Kao CC, Remple MS, D’Haese $\mathrm{PF}$, et al: Customized, miniature rapid-prototype stereotactic frames for use in deep brain stimulator surgery: initial clinical methodology and experience from 263 patients from 2002 to 2008. Stereotact Funct Neurosurg 89:34-41, 2011 
13. Mazziotta JC, Engel J Jr: The use and impact of positron computed tomography scanning in epilepsy. Epilepsia 25 (Suppl 2):S86-S104, 1984

14. Mehta AD, Labar D, Dean A, Harden C, Hosain S, Pak J, et al: Frameless stereotactic placement of depth electrodes in epilepsy surgery. J Neurosurg 102:1040-1045, 2005

15. Mullin JP, Shriver M, Alomar S, Najm I, Bulacio J, Chauvel $\mathrm{P}$, et al: Is SEEG safe? A systematic review and meta-analysis of stereo-electroencephalography-related complications. Epilepsia 57:386-401, 2016

16. Munari C, Hoffmann D, Francione S, Kahane P, Tassi L, Lo Russo G, et al: Stereo-electroencephalography methodology: advantages and limits. Acta Neurol Scand Suppl 152:5669, 1994

17. Nowell M, Rodionov R, Diehl B, Wehner T, Zombori G, Kinghorn J, et al: A novel method for implementation of frameless stereoEEG in epilepsy surgery. Neurosurgery $\mathbf{1 0}$ (Suppl 4):525-534, 2014

18. Pallavaram S, D'Haese PF, Lake W, Konrad PE, Dawant BM, Neimat JS: Fully automated targeting using nonrigid image registration matches accuracy and exceeds precision of best manual approaches to subthalamic deep brain stimulation targeting in Parkinson disease. Neurosurgery 76:756-765, 2015

19. Roessler K, Sommer B, Merkel A, Rampp S, Gollwitzer S, Hamer HM, et al: A frameless stereotactic implantation technique for depth electrodes in refractory epilepsy using intraoperative magnetic resonance imaging. World Neurosurg 94:206-210, 2016

\section{Disclosures}

Drs. Dawant, Pallavaram, and Konrad report holding equity in Neurotargeting, LLC, which produces the planning software used in this study under a licensing agreement with Vanderbilt University. Dr. Neimat reports a consultant relationship with FHC.

\section{Author Contributions}

Conception and design: Dewan, Pallavaram, Naftel. Acquisition of data: Shults, Hale, Sukul, Konrad, Yu, Neimat. Analysis and interpretation of data: Dewan, Shults, Rodriguez, Dawant. Drafting the article: Dewan, Naftel. Critically revising the article: Dewan, Hale, Sukul, Englot, Konrad, Yu, Neimat, Pallavaram. Reviewed submitted version of manuscript: all authors. Approved the final version of the manuscript on behalf of all authors: Dewan. Statistical analysis: Dewan. Study supervision: Dewan, Pallavaram, Naftel.

\section{Correspondence}

Michael C. Dewan, Department of Neurological Surgery, Vanderbilt University Medical Center, T-4224 Medical Center North, Nashville, TN 37232-2380. email: michael.dewan@vanderbilt.edu. 